\title{
Determination of the rock mass resistance index (GSI) based on image processing
}

\author{
Sorin Popescu ${ }^{1 *}$, and Ovidiu-Bogdan Tomuș ${ }^{1}$ \\ ${ }^{1}$ University of Petrosani, Department of Mechanical, Industrial and Transport Engineering, University \\ Street 20, Petrosani, Romania
}

\begin{abstract}
More and more often, and on an increasingly large scale, the geological resistance index (GSI) system is used for the design and practice of the mining process. The GSI, is a unique system for classifying the mass of rocks, linked to the parameters of rock strength and mass distortion, based on the generalized criteria of Hoek-Brown and MohrCoulomb. The GSI can be estimated using standard and in situ tables by direct surface observations in underground or surface mining. The GSI value provides a numerical representation of the overall Geotechnical quality of the rock mass. The method for determining GSI using photographic images of the in situ rock mass, with image processing technology, fractal theory and artificial neuronal network (ANN), is already known and successfully applied in several projects.
\end{abstract}

\section{Introduction}

In general, knowledge of the mechanical properties of the rock mass is a prerequisite for numerical simulation and the design of underground mining work.

Since the early 1990, a number of specialists including Hoek and Brown, 1997, Hoek and colab., 2005, Hoek and Marinos, 2007 [1-3]. They have proposed several methods to determine the resistance and deformation parameters of the rocks using the geological resistance Index (GSI). The standard GSI diagram shall take into account the qualitative surface condition and the blocking of a rock mass and shall be used to estimate a value between 0 and 100 representing the overall Geotechnical quality of the rock mass (Figure $1)$.

The best results can only be achieved through collaboration between experienced geologists and Geotechnical engineers. Quantifying GSI estimates can provide a means to reduce errors and inaccuracies in the classification of a rock mass by inexperienced practitioners. Several researchers have shown that GSI can be co-quantified using the rock quality Index (RQD-Rock quality design, proposed by Deere in 1964), the Q-rock quality Index, proposed by Barton and its contributors (1974), the RMR (Rock mass rating, proposed by Bieniawski (1974), or RSR (Rock structure rating) Proposed by Wickham (1972) [4].

\footnotetext{
*Corresponding author: sorin,popescu@toposystem.ro
} 


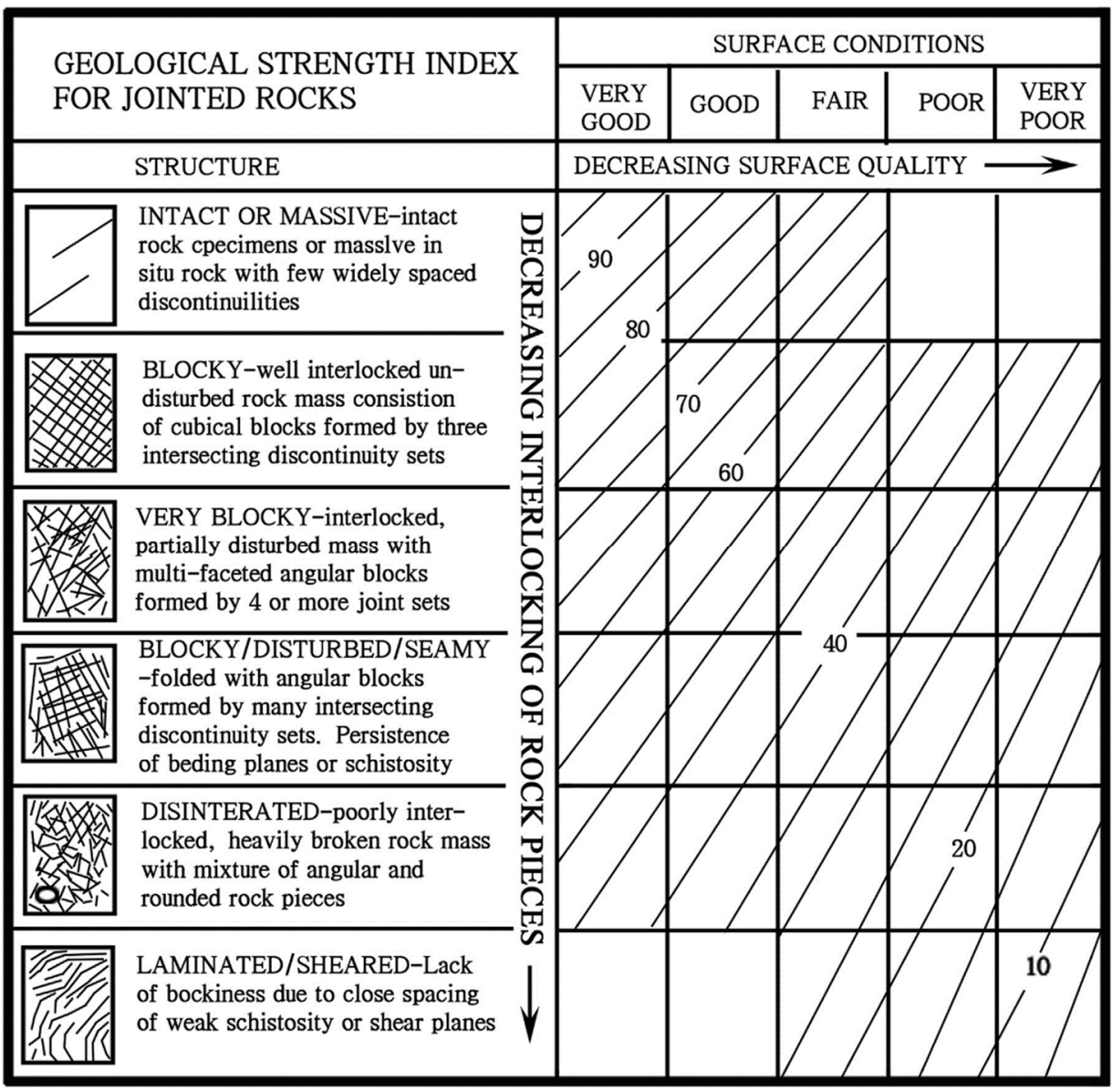

Fig. 1 Diagram for determining GSI (Hoek and Brown, 1997).

The shear strength parameters of a slip surface below a specific normal load can be determined using the material constants of the Hoek-Brown discontinuity criterion ( $\mathrm{mb}$ and s) depending on the GSI value. Also, the Tajani (2010) proposed a method of analysis of surface failure, which is based on uniaxial RC compression resistance of rock samples as well as mining and geological conditions, to determine the GSI values of a mass of rocks disturbed by the underground operation [3].

All the work mentioned above focused on quantifying the GSI graph to facilitate the use of the system, especially by inexperienced practitioners.

The Digital Rock mass rating (DRMR) developed by Monte (2004) uses basic procedures and calculations for image processing. The system incorporates information about fractures collected from a discontinuity tracking map (e.g. length, distance, roughness, or rock block volume) [5].

Digital surface mapping, as a practical tool for characterizing rock masses, can significantly reduce the time needed in the field and avoid possible interpretation errors.

The method of quantitative determination of GSI involves taking three steps:

-Detection of discontinuities (fractures, cracks, gaps, etc.) in two-dimensional (2D) photographs of the rock surface using image processing technology;

-Determination of the fractal dimension,

-Estimation of GSI using artificial Neuronal Network (ANN). 


\section{Detection of discontinuities on the surface of the rock mass by image processing}

The detailed steps for detecting these cracks are described below as follows:

\section{(1) convert the colour image to black and white}

In order to detect fractures on an excavated rock image, the contrast of the image must be analysed.

We convert the natural colour image to black and white using the grey level conversion function:

$$
g(x, y)=T[f(x, y)]=[R(x, y)+G(x, y)+B(x, y)] / 3
$$

where:

$\mathrm{g}(\mathrm{x}, \mathrm{y})$ is the output black and white image;

$\mathrm{f}(\mathrm{x}, \mathrm{y})$ is the entrance colour image;

$T$ is the operator of $f(x, y) ; R x, y), G x, y)$ and $B x, y)$.

\section{(2) smooth and sharpen}

To emphasize fractures, the image must be smoothed and sharpened using the appropriate "masks". Smoothing and sharpening can be achieved using the image enhancement technique using the following equation (William, 2007) [6]:

$$
g^{\prime}(x, y)=\int_{-\infty}^{+\infty} \int_{-\infty}^{+\infty} g(\xi, \eta) H(x-\xi, y-\eta) d \xi d \eta
$$

Where: $\mathrm{g}^{\prime}(\mathrm{x} . \mathrm{y})$ is the reduced or sharp image output, $\mathrm{g}(\xi, \eta)$ is the smooth or sharp image, and $H(x-\xi, y-\eta)$ is the matrix for smoothing or sharpening see the Figure 2.

$$
H(5,5)\left[\begin{array}{ccccc}
0 & \frac{1}{52} & \frac{1}{26} & \frac{1}{52} & 0 \\
\frac{1}{52} & \frac{1}{52} & \frac{1}{13} & \frac{1}{52} & \frac{1}{52} \\
\frac{1}{26} & \frac{1}{13} & \frac{1}{13} & \frac{1}{13} & \frac{1}{26} \\
\frac{1}{52} & \frac{1}{52} & \frac{1}{13} & \frac{1}{52} & \frac{1}{52} \\
0 & \frac{1}{52} & \frac{1}{26} & \frac{1}{52} & 0
\end{array}\right] H(3,3)\left[\begin{array}{ccc}
-1 & -1 & -1 \\
-1 & 8 & -1 \\
-1 & -1 & -1
\end{array}\right]
$$

Fig. 2. $H(5 X 5), H(3 X 3)$-the matrix for common masks for the smoothing and sharpening.

In EQ. (2), we need to choose the response to the H-pulse with low pass or high passthrough characteristics, respectively, for smoothing and sharpening. In detail, we use the 5 $\times 5$ low pass and $3 \times 3$ common masks for the smoothing and sharpening steps defined by matrix $\mathrm{H}(3 \times 3)$, see the figure 2 .

\section{(3) binary encoding}

For detection of emphasized fractures, a binary coding is carried out by discrimination analysis, so fractures and background of the image are separated from each other.

Since the brightness distributions of the different rock masses in the images are varied, we perform a binary coding by analysing discrimination to determine the threshold that sensibly separates pixels into two types, based on the concentration histogram. 


\section{(4) noise removal}

An image can be influenced by noise and interference from several sources, including electrical sensor noise, noise of photographic impurities and especially cracks induced by digging operations in mining (manual, shot or mechanical cutting). In general, the manual procedure eliminates explosion-induced cracks but other causes such as swelling or corrosion of rocks should be observed, while comparing the results of automatic detection with those of the in-situ survey.

\section{(5) detection of fractures}

After removing unnecessary noise, we detect any joints left on the binary image. In general, the background areas and lines (black or white) on the binary image can be easily found from the distribution of white pixels on the black background. For implementation, we use two major edge and line detection classes: Derived by order I and II. Details are explained in William (2007) [6].

In this way, we automatically collect the properties of the fracture system, such as fracture length and intensity, spacing and roughness, from discontinuity tracking images in an underground mining job on the 3 sides (left, right, and ceiling walls).

Image analysis for this purpose (determination of fractures), refers to the extraction of measurements, data or information from an image using automatic or semi-automatic methods.

Image analysis is distinguished from other types of image processing, such as encoding, restoration and enhancement, in that the end product of an image analysis system is usually a numeric output rather than an image.

Morphological image processing is a type of processing in which the spatial shape or structure of objects in an image change. Dilation, erosion and skeletisation are three fundamental morphological operations. With the expansion, an object increases evenly in space, while with erosion an object shrinks evenly. The skeletisation results in a representation of an object in the form of a walking stick.

Morphological operations of binary images are based on the geometric relationship or connectivity of pixels considered to be of the same class. In the binary image in Figure 3a, the black pixel ring divides the image into three segments: White pixels outside the ring, white pixels inside the ring, and the black pixels of the ring itself. The pixels in each segment are connected to each other. This connectivity concept is easy to understand for Figure $3 \mathrm{a}$, but ambiguity occurs when Figure $3 \mathrm{~b}$ is considered.

Do the black pixels define a ring or form four disconnected lines? The answers to these questions depend on the connectivity relationship between the pixels.

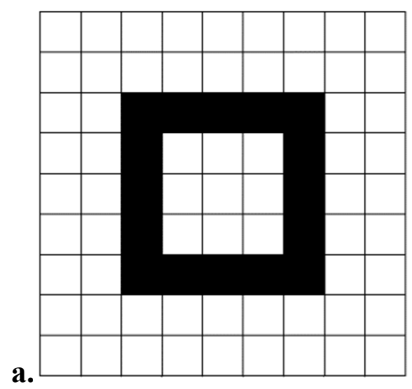

b.

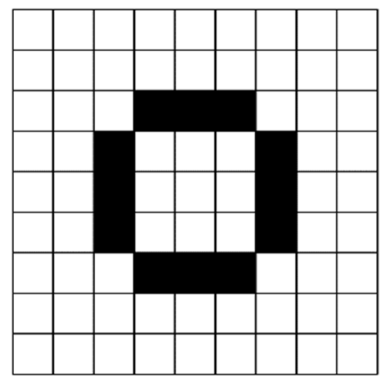

Fig. 3. Connectivity. a. Ring figures, b. Ambiguous figure

We consider the following model, table 1 : 
Table 1. Model of pixels.

$\begin{array}{lll}\mathrm{X}_{3} & \mathrm{X}_{2} & \mathrm{X}_{1} \\ \mathrm{X}_{4} & \mathrm{X} & \mathrm{X}_{0} \\ \mathrm{X}_{5} & \mathrm{X}_{6} & \mathrm{X}_{7}\end{array}$

$\begin{array}{cccc} & \text { NW } & \text { N } & \text { NE } \\ \text { or } & \text { W } & \text { X } & \text { E } \\ & \text { SW } & \text { S } & \text { SE }\end{array}$

In which a pixel with binary value, $X=0$ (white) or $X=1$ (black), is surrounded by its eight closest neighbours. X0, X1, X7. An alternative to labelling neighbours in the direction of the compass: north, North-East, etc.:

The X pixel has four connections if it is 1 logic and its neighbour is East, North, West or South (X0, X2, X4, X6) logic 1. Pixel X has eight connections to eight if it is 1 logic and if its neighbour in the north, north-east, etc. (X0, X1, X7) is a 1 logic.

The connectivity relationship between a central pixel and its eight neighbours can be measured by the sum of the weights of the link between the central pixel and each of its neighbours.

As defined with four connectivity, Figure $3 b$ has four segments of black line disconnected, but with the definition of eight connectivity, with a connected black pixel ring. If the black pixels have to be connected eight in a ring, a division of the white pixels into pixels that are internal and external to the ring may be expected. To eliminate this dilemma, we define 8 connectivity for the black object pixels and 4 connectivity for white background pixels. According to this definition, a string of black pixels is said to be minimal connected if the removal of any black pixel results in loss of black pixel connectivity.

\section{Fractal ownership of discontinuities on the surface of the rock}

In recent years, many researchers, including Jiang et al. (2006) and Alameda-Hernandez and colab. (2014) have investigated methods to determine the joint roughness coefficient (JRC) on the surface of the rock mass using the fractal dimension (D) $[7,8]$.

Odling, 1994, Fardin and colab, 2004, Nazarov and Nazarova, 2008, Wnuk and Yavari, 2008 and Wang and colab. (2015) have proposed the correlations between the fractal dimension and the joint properties on the surface of the rocks, such as the JRC [9-13].

On the basis of the above research, cracks in rock masses can in some cases be treated as statistical self-similar (fractal property) and can therefore be characterized using their fractal dimension.

The fractal dimension of a $2 \mathrm{D}$ rock mass area can be calculated with the following equation (Kulatilake and colab, 1997) [14]:

$$
D=\frac{\sum_{i=1}^{N}\left[\log _{10} N\left(r_{i}\right) \log _{10} r_{i}\right]}{\sum_{i=1}^{N}\left(\log _{10}^{2} r_{i}\right)}
$$

where: $\mathrm{r}_{\mathrm{i}}$ is the length of the section and $\mathrm{N}\left(\mathrm{r}_{\mathrm{i}}\right)$ is the number of fractures in the section under analysis.

Since the cracks in the rock mass are generally on a 2D plane, it is natural to consider the problem using the $2 \mathrm{D}$ fractal rather than $3 \mathrm{D}$ model.

When determining the fractal dimension for fractures on the rock mass surface, the study area is divided into square subareas (mesh) with dimensions between 1/64 and 1/2.

First, we divide the image into $2 n(n=0-6)$ for the detection of standard surface fractures according to the classification of the rock mass given by Hoek and Brown (1997) [1], then determine the number of fractures by the size of the mesh network, and finally, we 
calculate the fractal dimension of 6 standard types of ground surfaces. The fractal dimensions are listed in table 2 .

Table 2. Fracture dimensions according to the classification of rocks.By Hoek and Brown

(1997).

\begin{tabular}{|c|c|}
\hline Standard surface area of rock mass & Fractal dimension \\
\hline 1 type & 0.99237 \\
\hline 2nd type & 1.36661 \\
\hline Type 3 & 1.51057 \\
\hline 4th type & 1.68454 \\
\hline 5th type & 1.71558 \\
\hline Type 6 & 1.59083 \\
\hline
\end{tabular}

As shown in table 2, we can see that types 2 to 6 standard rock masses have a clear 2D fractal dimension. However, the first type of standard surface has a fractal dimension of approximately 0,99 , which does not belong to the $2 \mathrm{D}$ surface. This is because the first type of standard surface has 1-2 sets of joints. This can be a very important issue. Table 2 can therefore be used as an alternative tool allowing the quantification of standard rock masses in the GSI graph given by Hoek and Brown (1997) [1].

\section{Predicting GSI via the artificial-neural Network}

The development of ANN, or more simply NN-neuronal networks, started as an attempt to understand the functioning of the human brain and to imitate its assessment capabilities, in other words, to be able to decide and act in uncertain conditions or even to deal with situations with limited past experience. ANN are mathematical models consisting of interconnected processing nodes (neurones) within a pre-specified topology (layers).

As shown in Figure 1 and Table 2, the GSI and the fractal dimension are represented using different values depending on the surface properties of the respective rocks. Therefore, the GSI is closely related to the fractal dimension, as stated in the literature (Fardin and colab., 2004, Jiang and colab., 2006, Alameda-Hernandez and colab. 2014) [7, $8,10]$.

ANN is widely used in engineering practice. For example, the back propagation ANN (BP) is used to predict the deflection module and GSI of the fractured rock mass (Lemy and Hadjigeorgiou, 2003, Sonmez and colab., 2006) [15-18].

BP networks consist of an input layer, one or more hidden layers, and an output layer. Each layer consists of different processing units (also called neurones) connected to the following layer units. A transfer function processes input data that reaches the appropriate neurone. To differentiate between processing units, the values called biases are entered into the transfer functions. These biases are called the "temperature" of a neuron.

During network training, data is processed over the network until it reaches the forward pass layer. In this layer, the output is compared to the GSI value (the "true" output) obtained from Figure 1. If the default error is exceeded, the process resumes (BP) by updating the individual weights of the connections and biases of the individual neurones. The input and output data are mainly represented as vectors called training pairs. The above process shall be repeated for all training pairs in the data set until the network error reaches a minimum threshold defined by an appropriate cost function. 
For the assessment of GSI we used BN ANP with 3 starters. On 3-layer BP network, the $\mathrm{y}_{\mathrm{k}}$ output signal is expressed as:

$$
y_{k}=f\left[\sum_{j=1}^{m} W_{k j}^{o} f\left(\sum_{i=1}^{n} W_{j i}^{h} x_{i}-\theta_{j}^{h}\right)-\theta_{k}^{o}\right]
$$

where: $\quad x_{i}$ is the entry vector of the $\operatorname{ANN}(i=1,2, \ldots, n)$;

$\mathrm{y}_{\mathrm{k}}$ is the ANN exit vector $(\mathrm{k}=1,2, \ldots, \mathrm{n})$;

$\mathrm{W}_{\mathrm{ji}}^{\mathrm{h}}$ the weight vector from the entrance to the hidden layer $(\mathrm{j}=1,2, \ldots, \mathrm{m})$;

$\mathrm{W}_{\mathrm{kj}}{ }^{\mathrm{a}}$ weight vector from the hidden layer to the output layer $(\mathrm{k}=1,2, \ldots, \mathrm{L})$,

$\theta_{\mathrm{j}}^{\mathrm{h}}$ and $\theta_{\mathrm{k}}{ }^{\mathrm{O}}$ are the biases of the hidden layers and of the output respectively;

$f(*)$ is the transfer function; and

$\mathrm{n}, \mathrm{m}$, and 1 are the neurones of the infeed and outfeed layers respectively.

Based on the GSI graph, we establish an ANN model, where the input neurones are the fractal dimension and surface condition index (i.e. roughness and water supply condition) and the output neurone is a GSI value (Fig. 4).

Thus, the surface status index is changed from zero (very low) to 100 (very good, i.e. smooth and very hard surface).

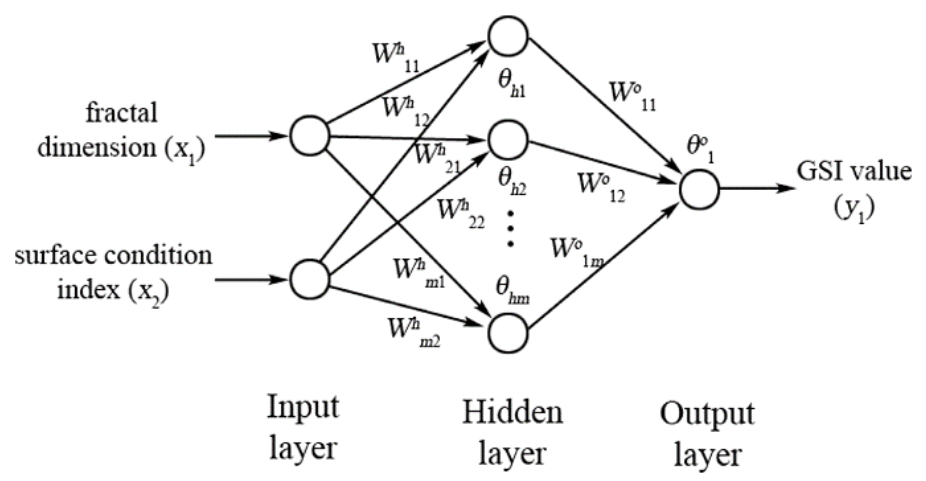

Fig. 4. The 3-layer ANN BP structure used in this study.

Here, the number of the hidden layer neurones is initially set to three and finally determined by the learning procedure to establish the most effective ANN structure. As a result of learning, the learning error is minimized by increasing the number of neurones of the hidden layer to 13 neurones when the highest accuracy of ANN learning is achieved.

In general, the output vector, $y_{k}$ of the output layer neurones, is not the same as the known output vector $y_{k}{ }^{*}$ (i.e. the GSI value obtained from Fig.1). The mean square error (Ek) of these vectors is made during the processing of the entry vector pair and can be calculated as follows:

$$
E_{k}=\frac{1}{2} \sum\left(y_{k}^{*}-y_{k}\right)^{2}
$$

The learning data set involves 310 data produced according to the rock mass classification and surface conditions of the rock mass in the GSI chart (Fig. (1) Hoek and Brown data (1997) and 40 of them are used to verify ANN accuracy [1].

After training of 1028 times, the mean square error of the ANN is reached up to the threshold error, and the relative error between the predicted GSI value of the ANN and the GSI value given in the GSI chart is less than 3,6\%. (Table 3). 
Table 3. Comparing GSI values according to the two methods

\begin{tabular}{|c|c|c|c|}
\hline \multirow{2}{*}{$\begin{array}{l}\text { CAS No: } \\
135\end{array}$} & \multicolumn{2}{|c|}{ GSI value } & \multirow{2}{*}{$\begin{array}{c}\text { Relative } \\
\text { error (\%) }\end{array}$} \\
\hline & Previous & New & \\
\hline 1 & 95 & 94 & 1.1 \\
\hline 2 & 97 & 98 & 1 \\
\hline 3 & 89 & 89 & 0 \\
\hline 4 & 86 & 88 & 2.3 \\
\hline 5 & 75 & 75 & 0 \\
\hline 6 & 82 & 81 & 1.2 \\
\hline 7 & 78 & 80 & 2.6 \\
\hline 8 & 92 & 94 & 2.2 \\
\hline 9 & 76 & 74 & 2.6 \\
\hline 10 & 79 & 81 & 2.5 \\
\hline 11 & 58 & 58 & 0 \\
\hline 12 & 73 & 72 & 1.4 \\
\hline 13 & 91 & 92 & 1.1 \\
\hline 14 & 62 & 61 & 1.6 \\
\hline
\end{tabular}

\begin{tabular}{|c|c|c|c|}
\hline \multirow{2}{*}{$\begin{array}{c}\text { CAS No: } \\
\text { 135 }\end{array}$} & \multicolumn{2}{|c|}{ GSI value } & $\begin{array}{c}\text { Relative } \\
\text { error (\%) }\end{array}$ \\
\cline { 2 - 3 } & Previous & New & \\
\hline 15 & 77 & 76 & 1.3 \\
\hline 16 & 59 & 58 & 1.7 \\
\hline 17 & 68 & 69 & 1.5 \\
\hline 18 & 77 & 78 & 1.3 \\
\hline 19 & 56 & 55 & 1.8 \\
\hline 20 & 65 & 66 & 1.5 \\
\hline 21 & 76 & 74 & 2.6 \\
\hline 22 & 86 & 86 & 0 \\
\hline 23 & 58 & 56 & 3.5 \\
\hline 24 & 76 & 75 & 1.3 \\
\hline 25 & 65 & 64 & 1.5 \\
\hline 26 & 93 & 95 & 2.2 \\
\hline 27 & 83 & 82 & 1.2 \\
\hline 28 & 66 & 68 & 3 \\
\hline
\end{tabular}

\begin{tabular}{|c|c|c|c|}
\hline \multirow{2}{*}{$\begin{array}{c}\text { CAS No: } \\
\text { 135 }\end{array}$} & \multicolumn{2}{|c|}{ GSI value } & \multirow{2}{*}{$\begin{array}{c}\text { Relative } \\
\text { error (\%) }\end{array}$} \\
\cline { 2 - 3 } & Previous & New & \\
\hline 29 & 49 & 50 & 2 \\
\hline 30 & 55 & 56 & 1.8 \\
\hline 31 & 70 & 72 & 2.9 \\
\hline 32 & 77 & 78 & 1.3 \\
\hline 33 & 97 & 96 & 1 \\
\hline 34 & 82 & 83 & 1.2 \\
\hline 35 & 84 & 85 & 1.2 \\
\hline 36 & 86 & 88 & 2.3 \\
\hline 37 & 74 & 73 & 1.4 \\
\hline 38 & 68 & 66 & 2.9 \\
\hline 39 & 85 & 85 & 0 \\
\hline 40 & 56 & 58 & 3.6 \\
\hline
\end{tabular}

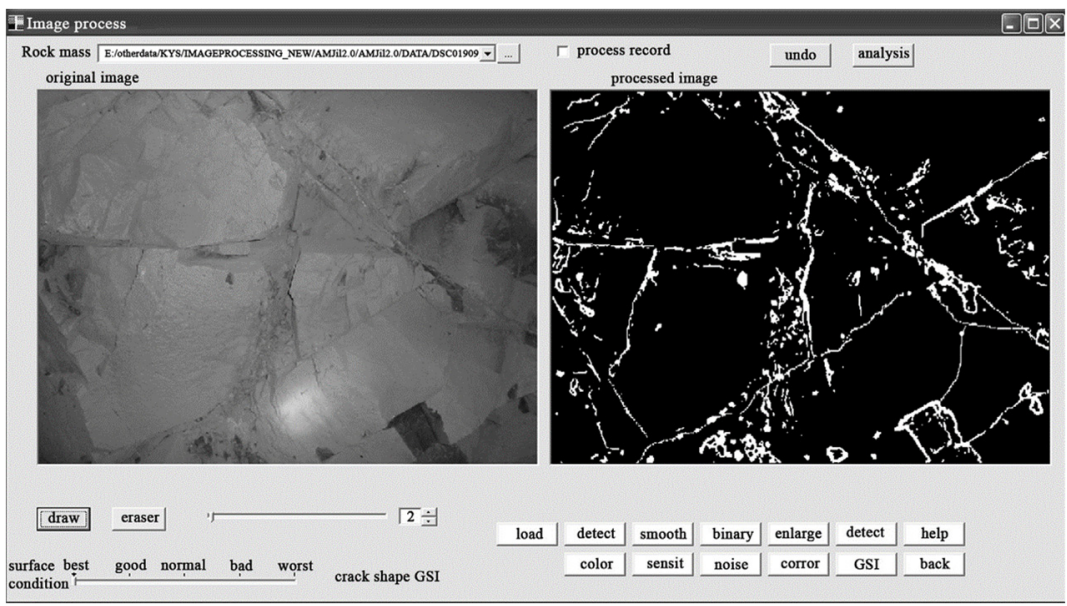

Fig. 5. Interface for GSI determination of the mass of articulated rocks.

Figure 5 provides the interface, encoded using built-in tools of MATLAB 7.0, such as image processing, fractal analysis and $\mathrm{ANN}$, based on the proposed method.

\section{Application example}

The applicability of the proposed method is checked by analysis of the stability of the works in a coal mine.

A main transport work from a coal mine is carried out into a relatively stable rock, which consists mainly of sandstone. The rock is easily degraded by one or two groups of fracture sets and the resistance to uniaxial compression of rock samples is approximately 88 $\mathrm{MPa}$, corresponding to Class I rocks according to the rock mass classification determined from the total evaluations (Bieniawski, 1978) [4]. 
The length of the mining work is about $800 \mathrm{~m}$ and the images are taken every $10 \mathrm{~m}$, a total of 240 images, for the left and right walls and the roof. Fig. 6 shows an example for some sections.

The results of determining the GSI value for each section are listed in Table 4. Thus, GSI values vary between 88 and 93 in the considered sections.

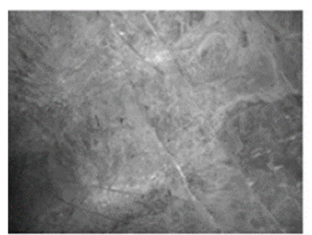

a)

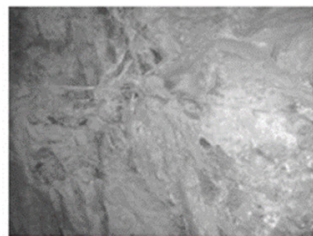

b)

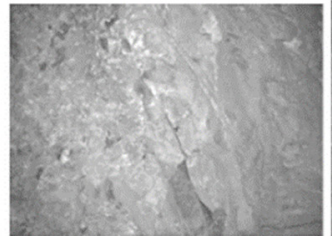

c)

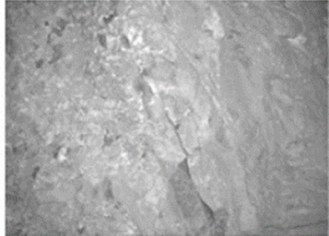

d)

Fig. 6. Images taken in the mining job.

Table 4. GSI values of the rock mass joined to the different sections.

\begin{tabular}{|c|c|c|c|c|c|c|c|c|c|c|c|c|}
\hline \multirow{2}{*}{ 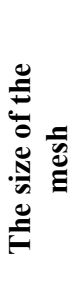 } & \multicolumn{4}{|c|}{$\begin{array}{c}\text { The number of joints in } \\
\text { each class }\end{array}$} & \multicolumn{4}{|c|}{ Fractal dimension } & \multicolumn{4}{|c|}{ GSI value } \\
\hline & 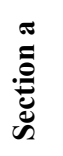 & 司 & 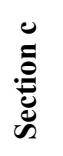 & 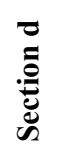 & 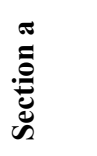 & 苛 & 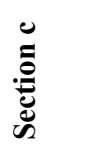 & تٓ & 䒕 & है & 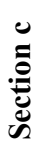 & 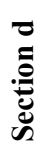 \\
\hline $2^{1}$ & 3 & 3 & 1 & 1 & \multirow{6}{*}{1.382} & \multirow{6}{*}{1.3505} & \multirow{6}{*}{1.225} & \multirow{6}{*}{1.1916} & \multirow{6}{*}{88} & \multirow{6}{*}{89} & \multirow{6}{*}{92} & \multirow{6}{*}{93} \\
\hline $2^{2}$ & 10 & 6 & 3 & 2 & & & & & & & & \\
\hline $2^{3}$ & 22 & 30 & 7 & 7 & & & & & & & & \\
\hline $2^{4}$ & 57 & 68 & 23 & 20 & & & & & & & & \\
\hline $2^{5}$ & 125 & 117 & 57 & 69 & & & & & & & & \\
\hline $2^{6}$ & 314 & 275 & 178 & 142 & & & & & & & & \\
\hline
\end{tabular}

\section{Conclusions}

The method for quantitative determination of GSI using in situ mass images leads to the following conclusions:

(1) the GSI mass diagram of the merged rocks given by Hoek and Brown (1997) can be quantified by combining image processing, fractal theory and ANN. In particular, the fractal dimension in Table 2 can be used as an alternative tool for quantifying standard rock masses in the GSI diagram.

(2) the proposed method is comparatively objective and can easily be used in engineering practice for underground and surface mining.

(3) this method should be further improved so that it can be applied to loose and soft rocks. 


\section{References}

1. E. Hoek, E. T. Brown, International Journal of Rock Mechanics and Mining Sciences, 34 (8), $1165 \mathrm{e} 86,(1997)$

2. E. Hoek, P. G. Marinos, V. P. Marinos, International Journal of Rock Mechanics and Mining Science 42 (2), 277e85, (2005)

3. E. Hoek, P. Marinos, Soils and Rocks 1e8, 2, (2007)

4. Bieniawski Z.T., Engineering rock mass classification, (Wiley, New York, 1989)

5. J.M. Monte, Rock mass characterization using laser scanning and digital imaging data collection techniques, MSc Thesis, Tucson, University of Arizona, USA, (2004)

6. $\quad$ K. P. William, Digital image processing, 4th ed. (John Wiley and Sons, Inc., 2007)

7. Y.J. Jiang, B. Li, Y. Tanabashi, International Journal of Rock Mechanics and Mining Sciences, 43(6):837e46 (2006)

8. Alameda-Hernandez P, Jiménez-Perálvarez J, Palenzuela JA, Hamdouni R, Irigaray C, Cabrerizo MA, Chacón J. Rock Mechanics and Rock Engineering 47(6):2047e60, (2014)

9. $\quad$ N.E. Odling, Rock Mechanics and Rock Engineering, 27(3):135e53 (1994)

10. N. Fardin, Q. Feng, O. Stephansson, International Journal of Rock Mechanics and Mining Sciences, 41 (2), 329e35, (2004)

11. L.A. Nazarov, L.A. Nazarova LA., Journal of Mining Science; 44(5):429e 38 (2008)

12. M.P. Wnuk, A. Yavari, Engineering Fracture Mechanics, 75(5):1127e42 (2008)

13. J.A. Wang, Y.X. Wang, Q.J. Cao, Y. Ju, L.T. Mao., International Journal of Rock Mechanics and Mining Sciences 2015;78:217e29

14. P. Kulatilake, R. Fiedler, B. Panda, Engineering Geology, 48(3e4):217e29 (1997)

15. J. Hadjigeorgiou, F. Lemy, P. Cote, X. Maldague, Rock Mechanics and Rock Engineering, 36 (2), $163 \mathrm{e} 79,(2003)$

16. H. Sonmez, C. Gokceoglu, H. A. Nefeslioglu, A. Kayabasi, International Journal of Rock Mechanics and Mining Sciences, 43 (2), 224e35, (2006)

17. M. Cai, P.K. Kaiser, Y. Tasaka, M. Minami, International Journal of Rock Mechanics and Mining Sciences 44 (2), 247e65, (2007)

18. H. Kunui, H. Eunchol, K. Kwangsong, Journal of Rock Mechanics and Geotechnical Engineering, 9, 702e708, (2017) 